\title{
Gamma ray heating rates due to chromium isotopes in stellar core during late stages of high mass stars $\left(>10 \mathrm{M}_{\odot}\right)$
}

\author{
Jameel-Un Nabi and Muhammad Fayaz \\ GIK Institute of Engineering Sciences and Technology, Topi 23640, Khyber Pakhtunkhwa, Pakistan
}

\begin{abstract}
Gamma ray heating rates are thought to play a crucial role during the pre-supernova stage of high mass stars. Gamma ray heating rates, due to $\beta^{ \pm}$-decay and electron (positron) capture on chromium isotopes, are calculated using proton-neutron quasiparticle random phase approximation theory. The electron capture significantly affects the lepton fraction $\left(\mathrm{Y}_{\mathrm{e}}\right)$ and accelerates the core contraction. The gamma rays emitted as a result of weak processes heat the core and tend to hinder the cooling and contraction due to electron capture and neutrino emission. The emitted gamma rays tend to produce enormous entropy and set the convection to play its role at this stage. The gamma heating rates, on ${ }^{50-60} \mathrm{Cr}$, are calculated for the density range $10<\rho\left(\mathrm{g}_{\mathrm{cm}} \mathrm{cm}^{-3}\right)<10^{11}$ and temperature range $10^{7}<\mathrm{T}(\mathrm{K})<3.0 \times 10^{10}$.
\end{abstract}

\section{Introduction}

Stars with masses greater than $10 \mathrm{M}_{\odot}$ proceed beyond oxygen burning and develop an electron degenerate iron core [1]. The electron degeneracy pressure supports the core against the gravity. The silicon burning in shell keeps growing the core towards the Chandrasekhar mass limit $\left(\sim 1.4 \mathrm{M}_{\odot}\right)$. At this stage electron degeneracy pressure cannot combat the enormous self gravity. The core becomes unstable and begins to collapse with a quarter of the speed of light [2]. The hot stellar core generates energetic gamma rays to initiate photodisintegration, which causes a complete collapse of the core. This way the gamma rays can remarkably affect the core growth, core collapse and core bounce during the last evolution phases of the stars. Also, the gamma heating rates cause convection to bring fresh material to the core and to continue the fusion process.

Weak interaction processes in massive stars provide the energy budget during the final stages of stellar life. Ref. [3] argues that neutrino cooling remarkably reduces the heating effect of gamma rays. Owing to core mass and its composition, these reactions can release 10 times more heat at densities $\sim 10^{11} \mathrm{~g} . \mathrm{cm}^{-3}$. The electron capture on various nuclei is sensitive to temperature [4]; so is the associated gamma heating. It is, therefore, worthwhile to calculate the associated gamma heating rates for the iron peak nuclei. During these processes, the stellar core stiffens to nuclear density and the collapsing core bounces against the nuclear matter. The parts of heavyelement core and outer shells are ejected into the interstellar medium [5]. The gamma heating, at this stage, may also contribute to revive the stalled shock, leading to explosive burning and convection.

The electron capture and $\beta$-decay rates, and associated neutrino-cooling and gamma heating rates, are sensitive to Gamow-Teller (GT) strength distributions. The neutrino-cooling occurs due to the production of (anti)neutrinos as a result of capture and decay processes. On the other hand, gamma ray heating results as the excited daughter nuclei (of capture and decay processes) return to their ground states. The GT transitions, in $f p$-shell nuclei, are crucially important in pre-supernova phases and the core collapse [6-9].

Isotopes of chromium play an important part in the pre-supernova evolution of massive stars. The measured data of GT strength in chromium isotopes is rather scarce. The decay of ${ }^{46} \mathrm{Cr}$ was studied by Ref. [10], by producing ${ }^{46} \mathrm{Cr}$ via the reaction ${ }^{32} \mathrm{~S}\left({ }^{16} \mathrm{O}, 2 n\right)$. Later Ref. [11] observed the $\beta$-decay of ${ }^{46} \mathrm{Cr}$ to the $1_{1}^{+}$state at 993 $\mathrm{keV}$ excitation energy in ${ }^{46} \mathrm{~V}$ at RIKEN. Ref. [12] did a ${ }^{50} \mathrm{Cr}\left({ }^{3} \mathrm{He}, \mathrm{t}\right){ }^{50} \mathrm{Mn}$ measurement up to $5 \mathrm{MeV}$ in daughter. A high resolution ${ }^{50} \mathrm{Cr}\left({ }^{3} \mathrm{He}, \mathrm{t}\right)^{50} \mathrm{Mn}$ measurement at an incident energy of $140 \mathrm{MeV} /$ nucleon and at $0^{0}$ was performed by Ref. [13] at RCNP, Japan. The authors measured $B(G T)^{-}$strength up to $12 \mathrm{MeV}$ in ${ }^{50} \mathrm{Mn}$. The simulation of astrophysical events (e.g. core-collapse supernovae), requires GT strength distribution for hundreds of nuclei. Therefore, theoretical estimates for GT strength distributions, microscopic in nature, are very much in demand.

The rate of change of $Y_{e}$ (lepton-to-baryon fraction) during pre-supernova evolution is one of the key inputs to generate a successful explosion. Ref. [14] found that the nuclei ${ }^{51-58} \mathrm{Cr}$, for electron capture, and ${ }^{53-57,59,60} \mathrm{Cr}$ for $\beta$-decay are of astrophysical significance in controlling $Y_{e}$ within the range $0.40 \leq Y_{e} \leq 0.50$. Ref. [15] found that ${ }^{50,51,53} \mathrm{Cr}$ were crucial for decreasing the $Y_{e}$ and ${ }^{53-56} \mathrm{Cr}$ played a significant role in increasing the $Y_{e}$ content of the stellar matter.

These studies motivated us to calculate the GT strength distributions and the associated gamma heating 
rates due to isotopes of chromium $\left({ }^{50-60} \mathrm{Cr}\right)$, both in electron capture and $\beta$-decay directions. The calculated GT distributions are compared with previous works for few even-even cases. We used the proton neutron quasiparticle random phase approximation (pn-QRPA) model to calculate the GT strength distributions and associated gamma heating rates in stellar matter. The formalism of our calculation is described briefly in the next section. We compare our results with other model calculations and measurements in Section 3.

\section{Model Description and Formalism}

A brief description of the pn-QRPA model is presented. For the detailed model formalism, we refer to Ref. [16]. The Hamiltonian of the pn-QRPA model is given by

$$
H^{Q R P A}=H^{S P}+V^{p a i r}+V_{G T}^{p h}+V_{G T}^{p p} .
$$

Wave functions and single particle energies were calculated using the Nilsson model. Pairing in nuclei was treated within the BCS approximation. The protonneutron residual interaction occurs through particle-hole (ph) and particle-particle (pp) channels. In our pn-QRPA model, these interactions were given a separable form as $V_{G T}^{p h}$ for the particle-hole Gamow-Teller force and $V_{G T}^{p p}$ for the particle-particle Gamow-Teller force. Our calculated GT strength distributions fulfilled the modelindependent Ikeda sum rule [17].

The other requisite model parameters are the Nilsson potential parameters, the pairing gaps, the nuclear quadrupole deformations, and the Q-values of the reactions. Nilsson potential parameters were adopted from Ref. [18] and the Nilsson oscillator constant was chosen as $\omega=41 A^{-1 / 3} \mathrm{MeV}$. The relation for pairing gaps was taken as: $\Delta_{p}=\Delta_{n}=12 / \sqrt{A} M e V$.

The nuclear quadrupole deformation parameters " $\beta$ " were taken from Ref. [19]. For even-even nuclei it was calculated using:

$$
\beta=\frac{4 \pi}{3 Z R_{0}^{2}}\left[B(E 2) \uparrow / e^{2}\right]^{1 / 2},
$$

where $B(E 2) \uparrow$ is the reduced electric quadrupole transition probability, $Z$ is the atomic number, and $R_{0}^{2}=0.0144 A^{2 / 3}$ barn. For remaining nuclei the deformation was taken from Ref. [20]. Q-values were taken from Ref. [21]. We used a model space of $7 \omega$ in our model calculation.

The basic formalism for calculation of stellar weak rates was adopted from the pioneering work of Fuller, Fowler and Newman (FFN) [6-9]. However, unlike the work of FFN, we calculated GT strength distributions from all parent excited states in a microscopic way. The weak decay rates of a transition from the $i^{\text {th }}$ parent to the $j^{\text {th }}$ daughter state is given by :

$$
\lambda_{i j}=\left(\frac{\ln 2}{D}\right)\left[f_{i j}\left(T, \rho, E_{f}\right)\right]\left[B(F)_{i j}+\left(g_{A} / g_{V}\right)^{2} B(G T)_{i j}\right] \text {. }
$$

The value of $\mathrm{D}=6143 \mathrm{~s}$ was taken from Ref. [22]. $B(F) i j$ denote the total reduced transition probability due to Fermi interaction and $B(G T) i j$ due to Gamow-Teller interactions. The value of $\left(\mathrm{g}_{\mathrm{A}} / \mathrm{g}_{\mathrm{V}}\right)=-1.2694$ (in Eq.3), was taken from [23]. The $f_{i j}$ are the phase space integrals which were calculated (in natural units $\hbar=\mathrm{m}_{\mathrm{e}}=\mathrm{c}=1$ ) for electron (upper signs) or positron (lower signs) emissions, using the relation:

$$
f_{i j}=\int_{1}^{w_{m}} w \sqrt{w^{2}-1}\left(w_{m}-w\right)^{2} F( \pm Z, w)(1-G) d w,
$$

the phase space for continuum positron (lower signs) or electron (upper signs) capture was calculated using:

$$
f_{i j}=\int_{w_{l}}^{\infty} w \sqrt{w^{2}-1}\left(w_{m}+w\right)^{2} F( \pm Z, w) G d w .
$$

In Eqs. (4) and (5) " $w$ " is the total energy (rest+kinetic) of the electron or positron, " $w_{l}$ " is the total threshold energy (rest+kinetic) for positron (or electron) capture and " $w_{m}$ " is the total $\beta$-decay energy. $F( \pm Z, w)$ are the Fermi functions and $G_{ \pm}$is the Fermi-Dirac distribution function for positrons (electrons).

The ratio of electron to baryon is given as :

$$
\rho Y_{e}=\frac{1}{\pi^{2} N_{A}}\left(\frac{m_{e} c}{{ }^{3}}\right)^{3}\left(G_{-}-G_{+}\right) p^{2} d p
$$

where $p=\left(w^{2}-1\right)^{1 / 2}$ is the electron or positron momentum, and Eq. (6) has the units of mole- $\mathrm{cm}^{-3}$. This equation was used for an iterative calculation of Fermi energies for selected values of $\rho Y_{e}$ and $T$.

The total gamma ray heating rate was calculated using the relation:

$$
\lambda^{\gamma}=\sum_{i j} P_{i} \lambda_{i j} E_{j}
$$

where " $P_{i}$ " is the probability of occupation of parent excited states and obey the Boltzmann distribution, $\lambda_{i j}$ gives the sum of the electron(positron) capture and positron(beta) decay rates, $\lambda_{i j}=\lambda_{i j}^{e c(p c)}+\lambda_{i j}^{p d(b d)}$, and $E_{j}$ is the energy of daughter's excited state.

\section{Results and Discussion}

In this section, we show our calculation of GT strength distributions and gamma heating rates for few even-even isotopes of chromium. The calculated GT strength in the $\beta$-decay direction for ${ }^{50} \mathrm{Cr}$ is shown in Fig. 1. The upper two panels show the measured GT distributions for ${ }^{50} \mathrm{Cr}$. The panel (Exp.1) shows the measurement of $\left({ }^{3} \mathrm{He}, \mathrm{t}\right)$ experiment up to $5 \mathrm{MeV}$ by Ref. [12]. The lower panel (Exp. 2) shows the $\mathrm{B}(\mathrm{GT})$. distribution up to $12 \mathrm{MeV}$ in daughter measured by Ref. [13]. The bottom panel of Fig. 1 further compares the pn-QRPA calculated GT strength distribution with the shell model calculation using the KB3G interaction, by Ref. [24]. It is noted that pn-QRPA calculated transitions are placed at lower energies and of bigger magnitude in daughter than the shell model results. It is inferred that the calculated GT distribution is in good conciliation with Exp. 2. The pnQRPA calculated GT. distribution in ${ }^{52} \mathrm{Cr}$ is compared with shell model calculation in Fig. 2. The shell model results spread over an energy range of 5-15 MeV in daughter. While the pn-QRPA places the energy centroid at low excitation energy of $5.41 \mathrm{MeV}$ in ${ }^{52} \mathrm{Mn}$. Fig. 3 shows a similar comparison of our GT. strength in ${ }^{54} \mathrm{Cr}$ with shell model results. The GT strength is 
placed at energy intervals of $2.5-10 \mathrm{MeV}$ in pn-QRPA model and 8-16 MeV in shell model calculation. The pnQRPA calculated ground and excited states GT strength distributions for ${ }^{50-60} \mathrm{Cr}$ are available and may be requested as ASCII files from the corresponding author.

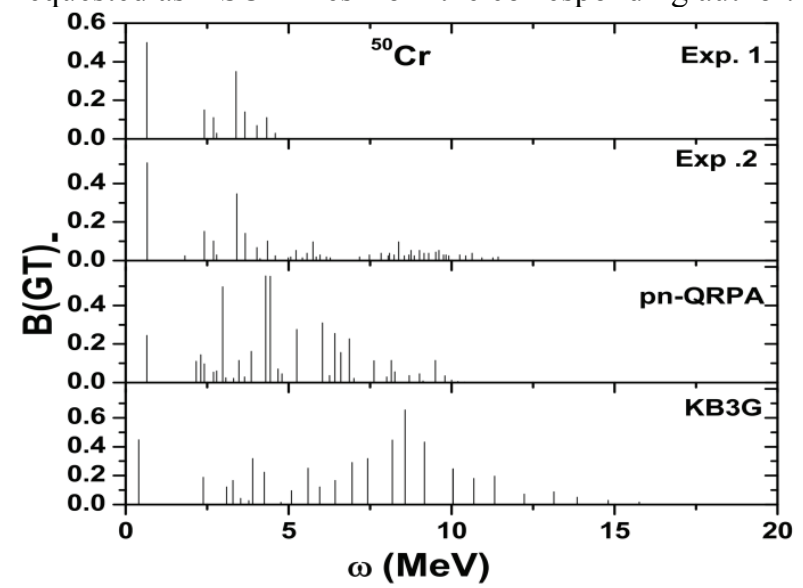

Figure 1 Comparison of B(GT)- strength distributions in ${ }^{50} \mathrm{Cr}$ with measured and calculated data. Exp. 1 shows measured distribution by [12], Exp. 2 by [13] while KB3G shows shell model calculation by [24]. $\omega$ represents excitation energy in ${ }^{50} \mathrm{Mn}$ in units of $\mathrm{MeV}$.

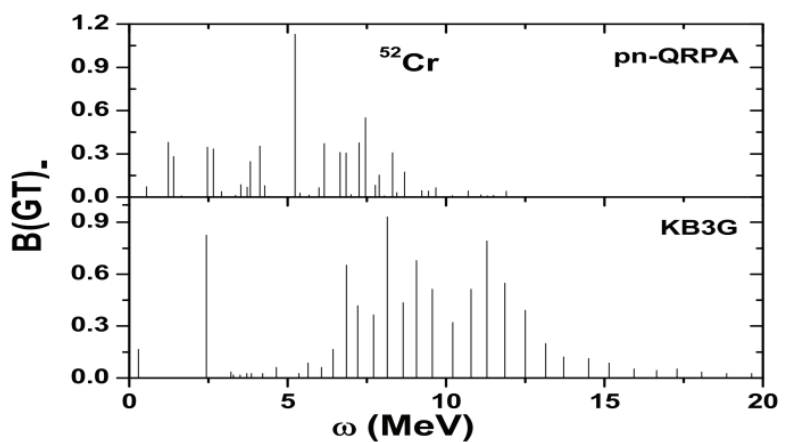

Figure 2 Comparison of pn-QRPA calculated B(GT). strength distributions in ${ }^{52} \mathrm{Cr}$ with shell model calculation [24]. $\omega$ represents excitation energy in ${ }^{52} \mathrm{Mn}$ in units of $\mathrm{MeV}$.

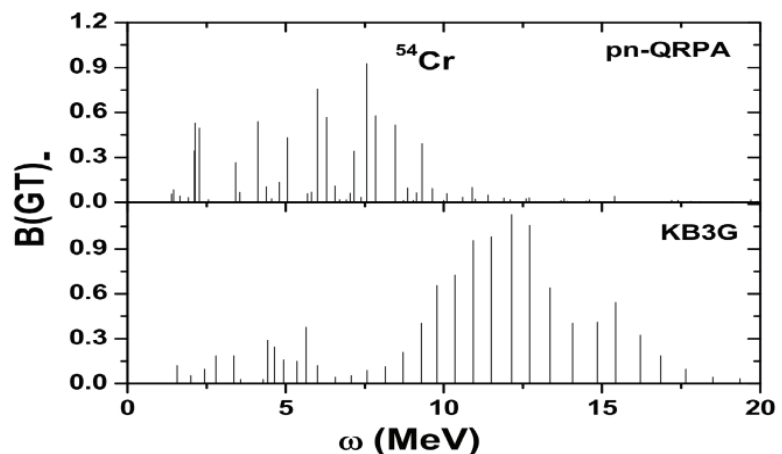

Figure 3 Comparison of pn-QRPA calculated B(GT). strength distribution in ${ }^{54} \mathrm{Cr}$ with shell model calculation [24]. $\omega$ represents excitation energy in ${ }^{54} \mathrm{Mn}$ in units of $\mathrm{MeV}$.

The gamma ray heating rates due to ${ }^{50} \mathrm{Cr},{ }^{52} \mathrm{Cr}$, and ${ }^{54} \mathrm{Cr}$ for selected densities $\left(1^{\text {st }}\right.$ column $)$ and temperatures $\left(2^{\text {nd }}\right.$ column) are shown in Table 1 . The value $1.00 \times 10^{-100}$ $\mathrm{MeV} . \mathrm{s}^{-1}$ means that the rates are negligibly small. These rates increase with temperature but those due to electron capture increase at faster rate than those due to $\beta$-decay for densities 10 to $10^{7} \mathrm{~g} . \mathrm{cm}^{-3}$. However, at $10^{11} \mathrm{~g} . \mathrm{cm}^{-3}$, the rates due to electron capture dominate over $\beta$-decay by orders of magnitudes and show nearly constant trend with temperature. In case of ${ }^{54} \mathrm{Cr}$, the gamma heating rates at lower densities dominate due to $\beta$-decay and at higher density due to electron capture. Next, we present the gamma heating rates due to ${ }^{56} \mathrm{Cr},{ }^{58} \mathrm{Cr}$, and ${ }^{60} \mathrm{Cr}$ in Table 2. Here again, a similar trend is witnessed for all these isotopes of chromium.

The ASCII files of calculated gamma heating rates due to isotopes of chromium, ${ }^{50-60} \mathrm{Cr}$, are available on a fine temperature-density grid, suitable for interpolation process, and may be requested from the corresponding author. It is hoped that the calculated heating rates may contribute to a better understanding of the dynamics of core evolution. We are in a process of calculating the neutrino cooling rates due to isotopes of chromium in stellar matter and hope to soon report our findings.

Acknowledgement: J.-U. Nabi would like to acknowledge the support of the Higher Education Commission Pakistan through the HEC Project No. 5557/KPK/NRPU/R\&D/HEC/2016, Pakistan Science Foundation through the project PSF-TUBITAK/KPGIKI (02) and TUBITAK project code 22211059B211700192.

\section{References}

1. R. Buras, M. Rampp, H.T. Janka, K. Kifonidis, Phys. Rev. Lett., 90(24), 241101(2003).

2. J. José, C. Iliadis, Rep. Prog. Phys., 74(9), 096901 (2011).

3. S. Gupta, E.F. Brown, H. Schatz, P. Möller, K.-L. Kratz, Astrophys. J. 662(2), 1188 (2007).

4. P.G. Giannaka, T.S. Kosmas, Adv. High Energy Phys. 2015 (2015).

5. H.T. Janka, K. Langanke, A. Marek, G. Martínez-Pinedo, B. Müller, Phys. Rep., 442(1), 38(2007).

6. G.M. Fuller, W.A. Fowler, M.J. Newman, Astrophys. J. Suppl. Ser. 42, 447 (1980)

7. G.M. Fuller, W.A. Fowler, M.J. Newman, Astrophys. J. Suppl. Ser. 48, 279 (1982).

8. G.M. Fuller, W.A. Fowler, M.J. Newman, Astrophys. J. 252, 715 (1982).

9. G.M. Fuller, W.A. Fowler, M.J. Newman, Astrophys. J. 293, 1 (1985).

10. J. Zioni, A.A. Jaffe, E. Friedman, N. Haik, R. Schectman, D. Nir, Nucl. Phys. A 181, 465 (1972).

11. T.K. Onishi et al., Phys. Rev. C 72, 024308 (2005).

12. Y. Fujita, B. Rubio, W. Gelletly, Prog. Part. Nucl. Phys. 66, 549 (2011).

13. T. Adachi et al., Nucl. Phys. A 778, 70c (2007).

14. M.B. Aufderheide, I. Fushiki, S.E. Woosley, Astrophys. J. Suppl. 91, 389 (1994).

15. A. Heger, S.E. Woosley, G. Martínez-Pinedo, K. Langanke, Astrophys. J. 560, 307 (2001).

16. J. -U. Nabi, H. V. Klapdor-Kleingrothaus, At. Data Nucl. Data Tables 71, 149 (1999).

17. I. Ikeda, Prog. Theor. Phys., 31, 434 (1964).

18. S. G. Nilsson, Mat. Fys. Medd. Dan. Vid. Selsk 29, 16 (1955).

19. S. Raman, C. W. Nestor, P. Tikkanen, At. Data Nucl. Data Tables 78(1), 1 (2001).

20. P. Möller A. J. Sierk, T. Ichikawa, H. Sagawa, At. Data Nucl. Data Tables 109, 1 (2016).

21. M. Wang, G. Audi, A. H. Wapstra, F. G. Kondev, M. MacCormick, X. Xu, B. Pfeiffer, Chin. Phys. C, 36, 1603 (2012). 
22. J. C. Hardy, I. S. Towner, Phys. Rev. C 79(5), 055502 (2009).

23. K. Nakamura (Particle Data Group), J. Phys. G: Nucl. Part. Phys. 37(7A), 075021 (2010).
24. I. Petermann, G. Martinez-Pinedo, K. Langanke, E. Caurier, Eur. Phys. J. A 34, 319 (2007)

Table 1 Calculated gamma heating rates (in $\mathrm{MeV} \cdot \mathrm{s}^{-1}$ ) due to weak rates on ${ }^{50} \mathrm{Cr},{ }^{52} \mathrm{Cr}$, and ${ }^{54} \mathrm{Cr}$ at selected densities and temperatures. The term $\lambda_{\gamma}^{p c+e d}$ is for gamma heating rates due to positron capture and $\beta^{-}$decay while $\lambda_{\gamma}^{e c+p d}$ is for gamma heating rates due to electron capture and positron decay.

\begin{tabular}{|c|c|c|c|c|c|c|c|}
\hline \multirow{2}{*}{$\rho\left(\mathrm{g} \mathrm{cm}^{-3}\right)$} & \multirow{2}{*}{$\mathrm{T}(\mathrm{K})$} & \multicolumn{2}{|c|}{${ }^{50} \mathrm{Cr}$} & \multicolumn{2}{|c|}{${ }^{52} \mathrm{Cr}$} & \multicolumn{2}{|c|}{${ }^{54} \mathrm{Cr}$} \\
\hline & & $\lambda_{\gamma}^{p c+e d}$ & $\lambda_{\gamma}^{e c+p d}$ & $\lambda_{\gamma}^{p c+e d}$ & $\lambda_{\gamma}^{e c+p d}$ & $\lambda_{\gamma}^{p c+e d}$ & $\lambda_{\gamma}^{e c+p d}$ \\
\hline \multirow{5}{*}{$1.00 \times 10^{1}$} & $1.00 \times 10^{7}$ & $1.00 \times 10^{-100}$ & $1.00 \times 10^{-100}$ & $1.00 \times 10^{-100}$ & $1.00 \times 10^{-100}$ & $1.00 \times 10^{-100}$ & $1.00 \times 10^{-100}$ \\
\hline & $1.00 \times 10^{8}$ & $1.00 \times 10^{-100}$ & $5.02 \times 10^{-67}$ & $1.00 \times 10^{-100}$ & $1.00 \times 10^{-100}$ & $1.00 \times 10^{-100}$ & $1.00 \times 10^{-100}$ \\
\hline & $1.00 \times 10^{9}$ & $1.13 \times 10^{-44}$ & $3.57 \times 10^{-15}$ & $1.38 \times 10^{-31}$ & $1.51 \times 10^{-29}$ & $1.19 \times 10^{-15}$ & $4.33 \times 10^{-43}$ \\
\hline & $1.00 \times 10^{10}$ & $2.45 \times 10^{-4}$ & $6.47 \times 10^{-2}$ & $3.98 \times 10^{-3}$ & $8.20 \times 10^{-3}$ & $5.89 \times 10^{-2}$ & $8.71 \times 10^{-4}$ \\
\hline & $3.00 \times 10^{10}$ & $1.42 \times 10^{1}$ & $2.94 \times 10^{2}$ & $5.96 \times 10^{1}$ & $1.59 \times 10^{2}$ & $1.72 \times 10^{2}$ & $1.10 \times 10^{2}$ \\
\hline \multirow{5}{*}{$1.00 \times 10^{4}$} & $1.00 \times 10^{7}$ & $1.00 \times 10^{-100}$ & $1.00 \times 10^{-100}$ & $1.00 \times 10^{-100}$ & $1.00 \times 10^{-100}$ & $1.00 \times 10^{-100}$ & $1.00 \times 10^{-100}$ \\
\hline & $1.00 \times 10^{8}$ & $1.00 \times 10^{-100}$ & $7.50 \times 10^{-64}$ & $1.00 \times 10^{-100}$ & $1.00 \times 10^{-100}$ & $1.00 \times 10^{-100}$ & $1.00 \times 10^{-100}$ \\
\hline & $1.00 \times 10^{9}$ & $1.01 \times 10^{-45}$ & $4.01 \times 10^{-14}$ & $1.70 \times 10^{-32}$ & $1.69 \times 10^{-28}$ & $1.06 \times 10^{-16}$ & $4.84 \times 10^{-42}$ \\
\hline & $1.00 \times 10^{10}$ & $2.45 \times 10^{-4}$ & $6.47 \times 10^{-2}$ & $3.98 \times 10^{-3}$ & $8.22 \times 10^{-3}$ & $5.89 \times 10^{-2}$ & $8.71 \times 10^{-4}$ \\
\hline & $3.00 \times 10^{10}$ & $1.42 \times 10^{1}$ & $2.95 \times 10^{2}$ & $5.96 \times 10^{1}$ & $1.59 \times 10^{2}$ & $1.72 \times 10^{2}$ & $1.10 \times 10^{2}$ \\
\hline \multirow{5}{*}{$1.00 \times 10^{7}$} & $1.00 \times 10^{7}$ & $1.00 \times 10^{-100}$ & $1.00 \times 10^{-100}$ & $1.00 \times 10^{-100}$ & $1.00 \times 10^{-100}$ & $1.00 \times 10^{-100}$ & $1.00 \times 10^{-100}$ \\
\hline & $1.00 \times 10^{8}$ & $1.00 \times 10^{-100}$ & $2.93 \times 10^{-28}$ & $1.00 \times 10^{-100}$ & $1.00 \times 10^{-100}$ & $1.00 \times 10^{-100}$ & $1.00 \times 10^{-100}$ \\
\hline & $1.00 \times 10^{9}$ & $5.83 \times 10^{-50}$ & $3.99 \times 10^{-9}$ & $1.95 \times 10^{-35}$ & $1.53 \times 10^{-23}$ & $1.21 \times 10^{-21}$ & $4.82 \times 10^{-37}$ \\
\hline & $1.00 \times 10^{10}$ & $1.95 \times 10^{-4}$ & $8.09 \times 10^{-2}$ & $3.18 \times 10^{-3}$ & $1.03 \times 10^{-2}$ & $4.76 \times 10^{-2}$ & $1.09 \times 10^{-3}$ \\
\hline & $3.00 \times 10^{10}$ & $1.41 \times 10^{1}$ & $2.97 \times 10^{2}$ & $5.92 \times 10^{1}$ & $1.60 \times 10^{2}$ & $1.71 \times 10^{2}$ & $1.11 \times 10^{2}$ \\
\hline \multirow{5}{*}{$1.00 \times 10^{11}$} & $1.00 \times 10^{7}$ & $1.00 \times 10^{-100}$ & $1.37 \times 10^{5}$ & $1.00 \times 10^{-100}$ & $8.57 \times 10^{4}$ & $1.00 \times 10^{-100}$ & $1.68 \times 10^{4}$ \\
\hline & $1.00 \times 10^{8}$ & $1.00 \times 10^{-100}$ & $1.36 \times 10^{5}$ & $1.00 \times 10^{-100}$ & $8.54 \times 10^{4}$ & $1.00 \times 10^{-100}$ & $1.68 \times 10^{4}$ \\
\hline & $1.00 \times 10^{9}$ & $1.00 \times 10^{-100}$ & $1.36 \times 10^{5}$ & $1.00 \times 10^{-100}$ & $8.55 \times 10^{4}$ & $1.00 \times 10^{-100}$ & $1.67 \times 10^{4}$ \\
\hline & $1.00 \times 10^{10}$ & $2.40 \times 10^{-16}$ & $1.44 \times 10^{5}$ & $4.16 \times 10^{-15}$ & $9.68 \times 10^{4}$ & $1.75 \times 10^{-13}$ & $2.89 \times 10^{4}$ \\
\hline & $3.00 \times 10^{10}$ & $1.95 \times 10^{-3}$ & $2.36 \times 10^{5}$ & $8.20 \times 10^{-3}$ & $1.82 \times 10^{5}$ & $2.40 \times 10^{-2}$ & $1.65 \times 10^{5}$ \\
\hline
\end{tabular}

Table 2 Same as Table 3.1 but for ${ }^{56} \mathrm{Cr},{ }^{58} \mathrm{Cr}$, and ${ }^{60} \mathrm{Cr}$.

\begin{tabular}{|c|c|c|c|c|c|c|c|}
\hline \multirow[b]{2}{*}{$\rho\left(\mathrm{g} . \mathrm{cm}^{-3}\right)$} & \multirow[b]{2}{*}{$\mathrm{T}(\mathrm{K})$} & \multicolumn{2}{|c|}{${ }^{56} \mathrm{Cr}$} & \multicolumn{2}{|c|}{${ }^{58} \mathrm{Cr}$} & \multicolumn{2}{|c|}{${ }^{60} \mathrm{Cr}$} \\
\hline & & $\lambda_{\gamma}^{p c+e d}$ & $\lambda_{\gamma}^{e c+p d}$ & $\lambda_{\gamma}^{p c+e d}$ & $\lambda_{\gamma}^{e c+p d}$ & $\lambda_{\gamma}^{p c+e d}$ & $\lambda_{\gamma}^{e c+p d}$ \\
\hline \multirow{5}{*}{$1.00 \times 10^{1}$} & $1.00 \times 10^{7}$ & $3.42 \times 10^{-4}$ & $1.00 \times 10^{-100}$ & $9.40 \times 10^{-2}$ & $1.00 \times 10^{-100}$ & $2.03 \times 10^{0}$ & $1.00 \times 10^{-100}$ \\
\hline & $1.00 \times 10^{8}$ & $3.42 \times 10^{-4}$ & $1.00 \times 10^{-100}$ & $9.40 \times 10^{-2}$ & $1.00 \times 10^{-100}$ & $2.03 \times 10^{0}$ & $1.00 \times 10^{-100}$ \\
\hline & $1.00 \times 10^{9}$ & $3.42 \times 10^{-4}$ & $1.56 \times 10^{-53}$ & $9.40 \times 10^{-2}$ & $1.11 \times 10^{-64}$ & $2.03 \times 10^{0}$ & $1.26 \times 10^{-75}$ \\
\hline & $1.00 \times 10^{10}$ & $5.20 \times 10^{-1}$ & $4.32 \times 10^{-4}$ & $1.06 \times 10^{1}$ & $9.23 \times 10^{-5}$ & $7.23 \times 10^{1}$ & $7.64 \times 10^{-6}$ \\
\hline & $3.00 \times 10^{10}$ & $2.32 \times 10^{2}$ & $9.59 \times 10^{1}$ & $1.29 \times 10^{3}$ & $5.22 \times 10^{1}$ & $3.21 \times 10^{3}$ & $2.76 \times 10^{1}$ \\
\hline \multirow{5}{*}{$1.00 \times 10^{4}$} & $1.00 \times 10^{7}$ & $3.37 \times 10^{-4}$ & $1.00 \times 10^{-100}$ & $9.35 \times 10^{-2}$ & $1.00 \times 10^{-100}$ & $2.03 \times 10^{0}$ & $1.00 \times 10^{-100}$ \\
\hline & $1.00 \times 10^{8}$ & $3.38 \times 10^{-4}$ & $1.00 \times 10^{-100}$ & $9.38 \times 10^{-2}$ & $1.00 \times 10^{-100}$ & $2.03 \times 10^{0}$ & $1.00 \times 10^{-100}$ \\
\hline & $1.00 \times 10^{9}$ & $3.41 \times 10^{-4}$ & $1.75 \times 10^{-52}$ & $9.38 \times 10^{-2}$ & $1.24 \times 10^{-63}$ & $2.03 \times 10^{0}$ & $1.26 \times 10^{-75}$ \\
\hline & $1.00 \times 10^{10}$ & $5.20 \times 10^{-1}$ & $4.32 \times 10^{-4}$ & $1.06 \times 10^{1}$ & $9.23 \times 10^{-5}$ & $7.23 \times 10^{1}$ & $1.66 \times 10^{-6}$ \\
\hline & $3.00 \times 10^{10}$ & $2.32 \times 10^{2}$ & $9.59 \times 10^{1}$ & $1.30 \times 10^{3}$ & $5.22 \times 10^{1}$ & $3.22 \times 10^{3}$ & $2.76 \times 10^{1}$ \\
\hline \multirow{5}{*}{$1.00 \times 10^{7}$} & $1.00 \times 10^{7}$ & $4.84 \times 10^{-5}$ & $1.00 \times 10^{-100}$ & $6.44 \times 10^{-2}$ & $1.00 \times 10^{-100}$ & $1.65 \times 10^{0}$ & $1.00 \times 10^{-100}$ \\
\hline & $1.00 \times 10^{8}$ & $4.84 \times 10^{-5}$ & $1.00 \times 10^{-100}$ & $6.43 \times 10^{-2}$ & $1.00 \times 10^{-100}$ & $1.65 \times 10^{0}$ & $1.00 \times 10^{-100}$ \\
\hline & $1.00 \times 10^{9}$ & $6.03 \times 10^{-5}$ & $1.75 \times 10^{-47}$ & $5.53 \times 10^{-2}$ & $1.24 \times 10^{-58}$ & $1.66 \times 10^{0}$ & $1.40 \times 10^{-69}$ \\
\hline & $1.00 \times 10^{10}$ & $4.35 \times 10^{-1}$ & $5.42 \times 10^{-4}$ & $1.00 \times 10^{1}$ & $1.16 \times 10^{-4}$ & $7.06 \times 10^{1}$ & $9.59 \times 10^{-6}$ \\
\hline & $3.00 \times 10^{10}$ & $2.30 \times 10^{2}$ & $9.68 \times 10^{1}$ & $1.29 \times 10^{3}$ & $5.27 \times 10^{1}$ & $3.20 \times 10^{3}$ & $2.79 \times 10^{1}$ \\
\hline \multirow{5}{*}{$1.00 \times 10^{11}$} & $1.00 \times 10^{7}$ & $1.00 \times 10^{-100}$ & $6.55 \times 10^{3}$ & $1.00 \times 10^{-100}$ & $2.06 \times 10^{3}$ & $1.00 \times 10^{-100}$ & $4.99 \times 10^{2}$ \\
\hline & $1.00 \times 10^{8}$ & $1.00 \times 10^{-100}$ & $6.56 \times 10^{3}$ & $1.00 \times 10^{-100}$ & $2.05 \times 10^{3}$ & $1.00 \times 10^{-100}$ & $5.01 \times 10^{2}$ \\
\hline & $1.00 \times 10^{9}$ & $1.00 \times 10^{-100}$ & $6.55 \times 10^{3}$ & $1.00 \times 10^{-100}$ & $2.05 \times 10^{3}$ & $1.23 \times 10^{-92}$ & $4.99 \times 10^{2}$ \\
\hline & $1.00 \times 10^{10}$ & $1.00 \times 10^{-11}$ & $1.75 \times 10^{4}$ & $1.26 \times 10^{-9}$ & $4.98 \times 10^{3}$ & $6.01 \times 10^{-8}$ & $2.31 \times 10^{3}$ \\
\hline & $3.00 \times 10^{10}$ & $3.36 \times 10^{-2}$ & $1.52 \times 10^{5}$ & $2.47 \times 10^{-1}$ & $8.41 \times 10^{4}$ & $1.52 \times 10^{0}$ & $5.69 \times 10^{5}$ \\
\hline
\end{tabular}

